Proceedings of the Edinburgh Mathematical Society (2002) 45, 547-548 (C)

DOI:10.1017/S0013091502000287 Printed in the United Kingdom

\title{
A DIMENSION-DEPENDENT MAXIMAL INEQUALITY
}

\author{
ROMAN SHVIDKOY \\ Department of Mathematics, University of Missouri-Columbia, \\ Columbia, MO 65211, USA (shvidkoy@math.missouri.edu)
}

(Received 26 February 2002)

\begin{abstract}
In this short note we show that $\sup \left\{\left\|M_{\nu}\right\|: \nu\right.$ is a measure on $\left.\mathbb{R}^{n}\right\}$, where $\left\|M_{\nu}\right\|$ denotes the centred Hardy-Littlewood maximal operator, depends exponentially on $n$.

Keywords: Hardy-Littlewood maximal operator; maximal inequality

AMS 2000 Mathematics subject classification: Primary 42B25
\end{abstract}

\section{Statement of the problem}

Let $\nu$ be a $\sigma$-finite measure on the Borel subsets of $\mathbb{R}^{n}$. Define the Hardy-Littlewood centred maximal operator associated with $\nu$ by

$$
M_{\nu} f(x)=\sup _{r>0}\left(\frac{1}{\nu\left(B_{r}(x)\right)}\right) \int_{B_{r}(x)}|f| \mathrm{d} \nu, \quad x \in \mathbb{R}^{n} .
$$

It was proved in $[\mathbf{1}, \mathbf{2}]$ that

$$
\left\|M_{\nu} f\right\|_{L_{p}\left(\mathbb{R}^{n}, \nu\right)} \leqslant C\|f\|_{L_{p}\left(\mathbb{R}^{n}, \nu\right)}, \quad 1<p<\infty,
$$

where $C$ does not depend on $\nu$. We present a simple construction showing that $C$ depends exponentially on $n$. This answers the question posed in $[\mathbf{2}, \mathbf{3}]$.

\section{Construction}

Claim 2.1. There is an absolute constant $\alpha>1$ such that one can find $\left[\alpha^{n}\right]$ points $x_{1}, x_{2}, \ldots, x_{\left[\alpha^{n}\right]}$ on the Euclidean sphere $S^{n-1}$ such that

$$
\left\|x_{i}-x_{j}\right\|>1, \quad i \neq j .
$$

The maximal value of $\alpha$ is immaterial. A simple argument based on volume estimates yields $\alpha \geqslant \mathrm{e}^{(\pi / 6)^{2} / 2}$.

Let us fix $x_{1}, x_{2}, \ldots, x_{\left[\alpha^{n}\right]}$ as in the claim and put

$$
\nu=\delta_{\{0\}}+\sum_{i} \delta_{\left\{x_{i}\right\}} .
$$


Define $f=\delta_{\{0\}}$. Then $\|f\|_{L_{p}\left(\mathbb{R}^{n}, \nu\right)}=1$. On the other hand,

$$
\left(M_{\nu} f\right)\left(x_{i}\right) \geqslant \frac{1}{\nu\left(B_{1}\left(x_{i}\right)\right)} \int_{B_{1}\left(x_{i}\right)}|f| \mathrm{d} \nu=\frac{1}{2}, \quad i=1, \ldots,\left[\alpha^{n}\right] .
$$

Hence, $\left\|M_{\nu} f\right\|_{L_{p}\left(\mathbb{R}^{n}, \nu\right)} \geqslant \frac{1}{2}\left[\alpha^{n}\right]$.

This is the end of the construction.

\section{References}

1. R. Fefferman, Strong differentiation with respect to measures, Am. J. Math. 103 (1981), $33-40$.

2. L. Grafakos And J. Kinnunen, Sharp inequalities for maximal functions associated with general measures, Proc. R. Soc. Edinb. A 128 (1998), 717-723.

3. I. E. Verbitsky, A dimension-free Carleson measure inequality, in Complex analysis, operators, and related topics, pp. 393-398 (Birkhäuser, Basel, 2000). 